\title{
Design of a Variant of Vascular Endothelial Growth Factor-A (VEGF-A) Antagonizing KDR/FIk-1 and Flt-1
}

\author{
William Leenders, Nicolette Lubsen, Margarethe van Altena, Matthias Clauss, \\ Martine Deckers, Clemens Löwik, Georg Breier, Dirk Ruiter, and Robert de Waal
}

Department of Pathology, University Medical Center Nijmegen; Department of Biochemistry (NL), Faculty of Science, University of Nijmegen, Nijmegen, The Netherlands; Max Planck Institute for Physiological and Clinical Research (MC, GB), Bad Nauheim, Germany; and Department of Endocrinology (MD, CL), Leids Universitair Medisch Center, Leiden, The Netherlands

SUMMARY: Because of its central role in pathological angiogenesis, vascular endothelial growth factor (VEGF) has become a major target for anti-angiogenic therapies. We report here the construction of a heterodimeric antagonistic VEGF variant (HD-VEGF). In this antagonist, binding domains for the VEGF-receptors KDR/FIk-1 and Flt-1 are present at one pole of the dimer, whereas the other pole carries domain swap mutations, which prevent binding to either receptor. As HD-VEGF can only bind to monomeric receptors, it does not lead to signal transduction. Moreover, it antagonizes VEGF and possibly other members of the VEGF family, which are KDR/FIk-1 and Flt-1 ligands. We show here that HD-VEGF is a potent inhibitor of VEGF-mediated proliferation and tissue factor induction in endothelial cell cultures, requiring only a 20 -fold and a 4-fold excess, respectively, to block the activity of wtVEGF completely. A 4-fold excess of HD-VEGF over wtVEGF was also sufficient to abrogate vascular permeability as determined in the Miles assay in vivo. Furthermore, HD-VEGF inhibited fetal bone angiogenesis in an ex vivo assay. Thus, HD-VEGF blocks KDR- and Flt-1-mediated VEGF activities that are crucial in the angiogenic process and is therefore a promising, multipotent compound in the treatment of angiogenesis-related diseases. (Lab Invest 2002, 82:473-481).

A ngiogenesis, the sprouting of new blood vessels from pre-existing ones, is involved in many pathophysiological conditions like wound healing, rheumatoid arthritis, diabetic retinopathy, and psoriasis (Folkman and Klagsbrun, 1987a; Hanahan and Folkman, 1996). The pivotal role of angiogenesis in tumor biology is widely recognized. Tumors depend on a vascular bed not only for supply of oxygen and nutrients, but also for the capacity to metastasize (Folkman and Klagsbrun, 1987b). Microvessel density correlates with poor prognosis in a number of solid tumor types (Takahashi et al, 1995; Viglietto et al, 1995) and, conversely, inhibition of angiogenesis in various animal tumor models leads to inhibition of tumor growth or even regression of tumors (Asano et al, 1995; Benjamin and Keshet, 1997; Borgström et al, 1996; Cheng et al, 1996; Kim et al, 1993; Leenders, 1998; Millauer et al, 1996). Because angiogenesis in the normal adult is restricted to the menstrual cycle, anti-angiogenic therapy can be considered as tumorspecific, with few, if any, possible side effects at distant sites in the body.

Vascular endothelial growth factor-A (VEGF-A) can stimulate a number of biological responses in endo-

Received November 27, 2001.

This work was supported by a grant from the Dutch Cancer Society (grant 95-010).

Address reprint requests to: Dr. William Leenders, University Medical Center Nijmegen, Department of Pathology, PO Box 9101, 6500 HB Nijmegen, The Netherlands.E-mail: w.leenders@pathol.azn.nl thelial cells such as proliferation, migration, vascular permeability (VP), and production of proteases and their receptors, creating the prime conditions under which angiogenesis can occur (Claffey et al, 1995; Pötgens et al, 1994). VEGF activities are mediated by two tyrosine-kinase receptors, Flt-1 and KDR/Flk-1, whereas neuropilin has been identified as an isoformspecific co-receptor (Neufeld et al, 1999; Soker et al, 1996). The roles of KDR/Flk-1 and Flt-1 in mediating the cellular responses to VEGF have not yet fully been elucidated. Knocking out either KDR/Flk-1 or Flt-1 leads to embryonic lethality although the underlying defects are different (Fong et al, 1995; Shalaby et al, 1995). In pathological angiogenesis, blocking KDR/ Flk-1 by a dominant-negative strategy or by anti-KDR antibodies is effective in abrogating angiogenesis in tumor xenograft models (Millauer et al, 1996; Witte et al, 1998). The role of Flt-1 in pathological angiogenesis is less clear. Flt-1 phosphorylation in adult endothelial cells is difficult to demonstrate. However, ribozymes that target Flt-1 are effective in halting angiogenesis (Weng and Usman, 2001), and overexpression of placenta growth factor (PIGF), a Flt-1 selective ligand, leads to marked tumor progression (Hiratsuka et al, 2001). In vitro, Flt-1 induces tissue factor expression and migration in endothelial cells (Clauss et al, 1996; Park et al, 1994; Shibuya, 2001) and monocytes (Barleon et al, 1996). An additional important role for Flt-1 in cancer is shown by the recent finding that via this receptor VEGF suppresses maturation of, and antigen presentation by, dendritic cells (DCs) thus 
leading to an impaired anti-tumor response by these cells (Gabrilovitch et al, 1998; Oyama et al, 1998). Blockade of Flt-1 might, therefore, result in attenuation of VEGF-induced immune suppression and a possible relief of immune escape by the tumor. Taken together, blockade of both VEGF receptors simultaneously is likely to be important when attempting to block angiogenesis.

VEGF is a member of a superfamily of homodimeric growth factors, encompassing platelet-derived growth factor (PDGF)-A and B, PIGF, VEGF-B, VEGF-C, VEGF-D, and VEGF-E (Achen et al, 1998; Andersson et al, 1995; Joukov et al, 1996; Meyer et al, 1999; Neufeld et al, 1999; Olofsson et al, 1996; Park et al, 1994). These factors share a common motif, the cystine knot, which consists of eight spatially conserved cysteines that are involved in intra- and intermolecular disulphide bonds. Two cysteines, the second and fourth in the knot, interact intermolecularly, leading to the antiparallel conformation of the dimer (Andersson et al, 1992; Pötgens et al, 1994). In PDGF, three exposed domains, loops 1 to 3 , are involved in receptor binding (Andersson et al, 1992; Andersson et al, 1995). Alanine-scanning mutagenesis in the corresponding domains in VEGF revealed that charged amino acid residues in two exposed domains of VEGF, in analogy to the PDGF situation called loop 2 and loop 3, are involved in binding to Flt-1 and KDR/Flk-1, respectively (Fuh et al, 1998; Keyt et al, 1996).

In the present work, we have exchanged loop 1, loop 2, and loop 3 of VEGF with the corresponding domains of PDGF-B. We show that homodimers of the loop 1-mutated molecule have wild-type affinity for the Flt-1 receptor but more than 100-fold reduced affinity for the KDR/Flk-1 receptor. Conversely, homodimers of the loop 2 mutated molecule had near wild-type affinity for KDR/FIk-1 but no affinity for Flt-1. Using this information, a heterodimeric VEGF variant (HDVEGF) was generated that has retained at one pole the ability to bind both VEGF receptors KDR/FIk-1 and Flt-1 but lost the ability to bind these receptors at the other pole of the dimer (Fig. 1A). This protein potently antagonizes the effect of wtVEGF in proliferation assays, tissue factor induction assay, the Miles VP assay, and in an ex vivo fetal bone vascular outgrowth assay.

\section{Results}

\section{Design and Construction of the Heterodimeric VEGF-Mutant}

The structural homology between PDGF and VEGF predicts that the exposed loops 1, 2, and 3 may be swapped between these proteins without affecting folding. Indeed, VEGF homodimers in which loop 1, loop 2, or loop 3 had been replaced by the corresponding loops of PDGF-B could be readily prepared in the baculovirus expression system (not shown). VEGF-L1 (in which loop 1 of VEGF had been substituted by loop 1 of PDGF-B) did not bind to predimerized KDR (as a KDR-Fc fusion protein consisting of the
A
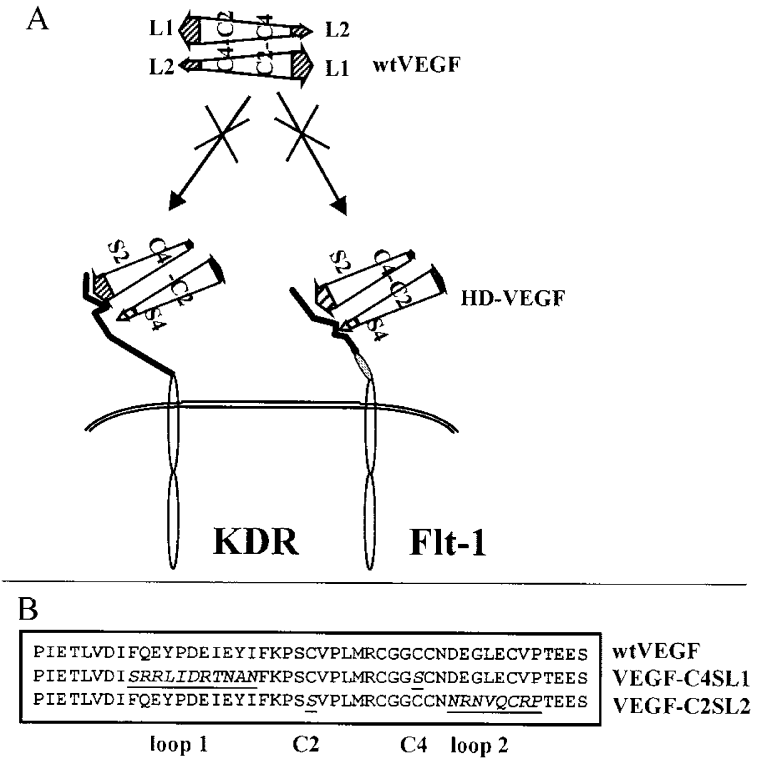

Figure 1.

A, Model of antagonism of a heterodimeric antagonistic VEGF variant (HDVEGF). Normally, wtVEGF dimerizes KDR receptors via binding domains involving loop 1 (L1) on opposite sides of the symmetrical dimer, whereas loop 2 (L2) is involved in binding Flt-1 receptors. HD-VEGF can bind monomeric receptors only and thus will not give rise to receptor (homo- or hetero-) dimerization and subsequent activation but will compete with wtVEGF for receptor binding. B, Amino acid sequences of the mutated regions. Underlined, italic sequences represent the receptor binding loops 1 and 2 from platelet-derived growth factor (PDGF)-B. Shown are amino acids 28 to 74 (numbering is according to the mature protein).

extracellular domain of KDR fused to the Fc region of human $\operatorname{lgG}$ ), although it did bind efficiently to Flt-1-Fc (Fig. 2A). This result was confirmed in receptor competition assays: VEGF-L1 1 competed for ${ }^{125}$ I-VEGF binding to Flt-1-expressing fibroblasts as efficiently as wtVEGF (Fig. 2C), whereas, based on the ED50 values, it had more than 50-fold reduced affinity for the KDR/FIk-1 receptor (Fig. 2B). Conversely, swapping loop 2 led to a 100 to 300-fold decrease of Flt-1 affinity (Fig. 2C), whereas this mutation had only a minor effect on KDR/Flk-1 binding in competition assays (Fig. 2B). Despite the affinity-decrease of VEGF-L2 for Flt-1 on 3T3 cells, we still found binding of VEGF-L2 to predimerized Flt-1-Fc (Fig. 2A) indicating the presence of a strong cooperativity in this interaction. Substitution of loop 3 in VEGF led to a 6 to 10 -fold reduction of affinity for KDR/Flk-1 in competition studies, whereas affinity for the Flt-1 receptor was not affected (not shown). As VEGF-L1 had lost affinity for KDR/ Flk-1, and VEGF-L2 had lost affinity for Flt-1, a VEGFL1/NEGF-L2 heterodimer would have one mutant pole, which cannot bind to either KDR/Flk-1 or Flt-1, and one wild-type pole, with wild-type affinity for both receptors (see Fig. 1A). To construct this heterodimer, the L1 mutation was combined with a C4S mutation in one subunit, whereas the $\mathrm{L} 2$ mutation was combined with a C2S mutation in the other subunit. These $\mathrm{C}$ to $\mathrm{S}$ mutations block homodimerization of the subunits but allow heterodimer formation when expressed by the same cell (Pötgens et al, 1994; see also Fig. 1A). As expected, SF9 insect cells secreted VEGF-C2SL2 and 

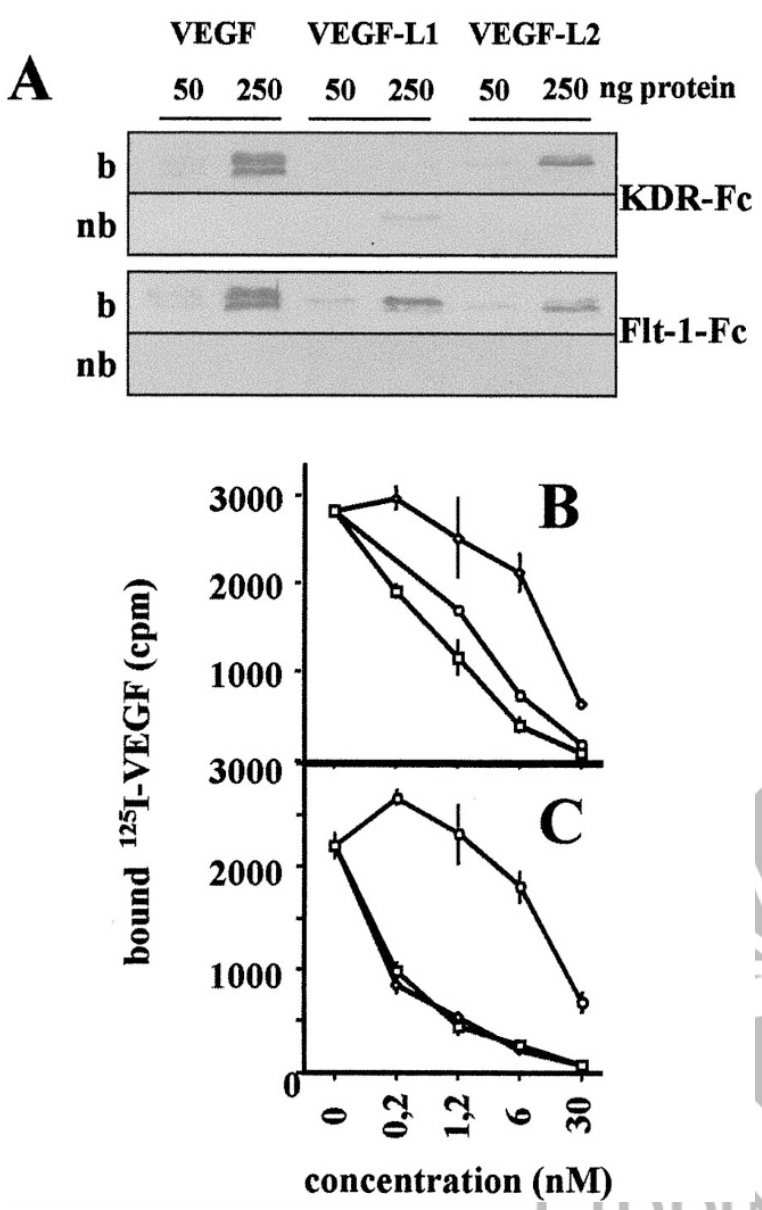

Figure 2.

Receptor binding of mutants of vascular endothelial growth factor (VEGF), containing domain swap mutations of loop 1 or loop 2. A, Binding of VEGF (mutants) to predimerized receptors as described in the text. Fifty or $250 \mathrm{ng}$ of VEGF (mutant) was allowed to bind to immobilized KDR-FC or Flt-1-Fc. Nonbound $(n b)$ and bound (b) proteins were subjected to electrophoresis through 12\% SDS-PAGE gels, blotted onto Hybond and immunostained with an anti-VEGF antibody. It is important to note that nonbound represents only $10 \%$ of the total unbound protein, due to gel loading limitations, leading to an underestimation of the true quantities. B and C, Competition by wtVEGF

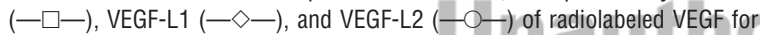
binding to $3 T 3$ cells stably expressing KDR (B) or Flt-1 (C). Procedures are described in the text.

VEGF-C4SL1 as monomers, whereas co-production of these HD-VEGF-constituents led to secretion of heterodimers (Fig. 3).

\section{Analysis of Activity of VEGF Mutants}

HD-VEGF and the homodimeric mutant constituent proteins were tested for their capacity to induce proliferation. VEGF-L1 and HD-VEGF were not able to induce proliferation in HUVEC, unlike VEGF-L2 (Fig. 4A). To test whether HD-VEGF could inhibit wtVEGF, we incubated HUVE cells with $10 \mathrm{ng} / \mathrm{ml}$ wtVEGF and increasing concentrations of HD-VEGF. A 20-fold excess of HD-VEGF over wtVEGF completely inhibited proliferation whereas addition of the composing subunits VEGF-C2SL2 or VEGF-C4SL1 alone had little or no effect (Fig. 4B).
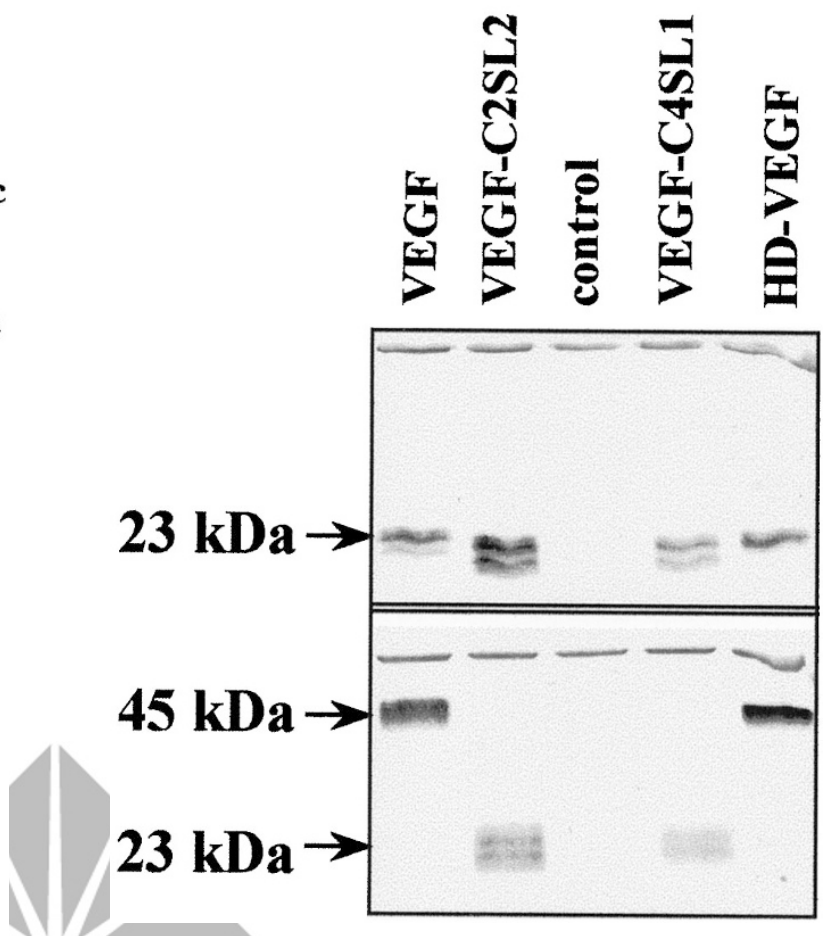

Figure 3.

Western blots of reducing (upper part) and nonreducing 12\% PAGE gels containing recombinant baculovirus-derived VEGF mutants. All proteins migrate at $\sim 23 \mathrm{kDa}$ on reducing gels (arrow). The doublet appearance is due to partial glycosylation (data not shown). Note that the subunits of HD-VEGF (VEGF-C2SL2 and VEGF-C4SL1) migrate as $\sim 23 \mathrm{kDa}$ monomers on nonreducing gels, whereas co-production of these subunits by SF9 cells leads to the 45 $\mathrm{kDa}$ dimer, indicating efficient heterodimerization of the subunit components. In the control lane, heparin-purified proteins from media, conditioned on mock-infected cells, were loaded.

We then tested these mutants for their ability to induce tissue factor (TF), as measured by the procoagulant assay. Also in this assay, VEGF-L1 and HDVEGF were unable to induce TF while VEGF-L2 showed a little activity at higher concentrations (data not shown). To test whether HD-VEGF also inhibited wtVEGF-induced TF-up-regulation, we activated HUVECs with $10 \mathrm{ng} / \mathrm{ml}$ wtVEGF in the presence of increasing concentrations of HD-VEGF. A 4-fold excess of HD-VEGF was already sufficient to block the activity of wtVEGF completely in this assay. This inhibition was specific for HD-VEGF, because VEGFC1S, an inactive control protein, had no such effect (Fig. 5).

One of the properties that distinguishes VEGF from other angiogenic factors such as basic fibroblast growth factor (bFGF), is its ability to induce vascular permeability (VP). We tested the activities of the different mutant proteins in the Miles VP assay. The VEGF-L1 homodimer showed no activity, whereas, although at higher concentrations than wtVEGF, VEGF-L2 was slightly active. Again, HD-VEGF was inactive (Fig. 6A). HD-VEGF inhibition of wtVEGFinduced VP is shown in Figure 6B. Injection of a 4-fold excess HD-VEGF over wtVEGF reproducibly led to a complete abrogation of extravasation of Evan's Blue. This inhibitory effect was again specific for HD-VEGF, 

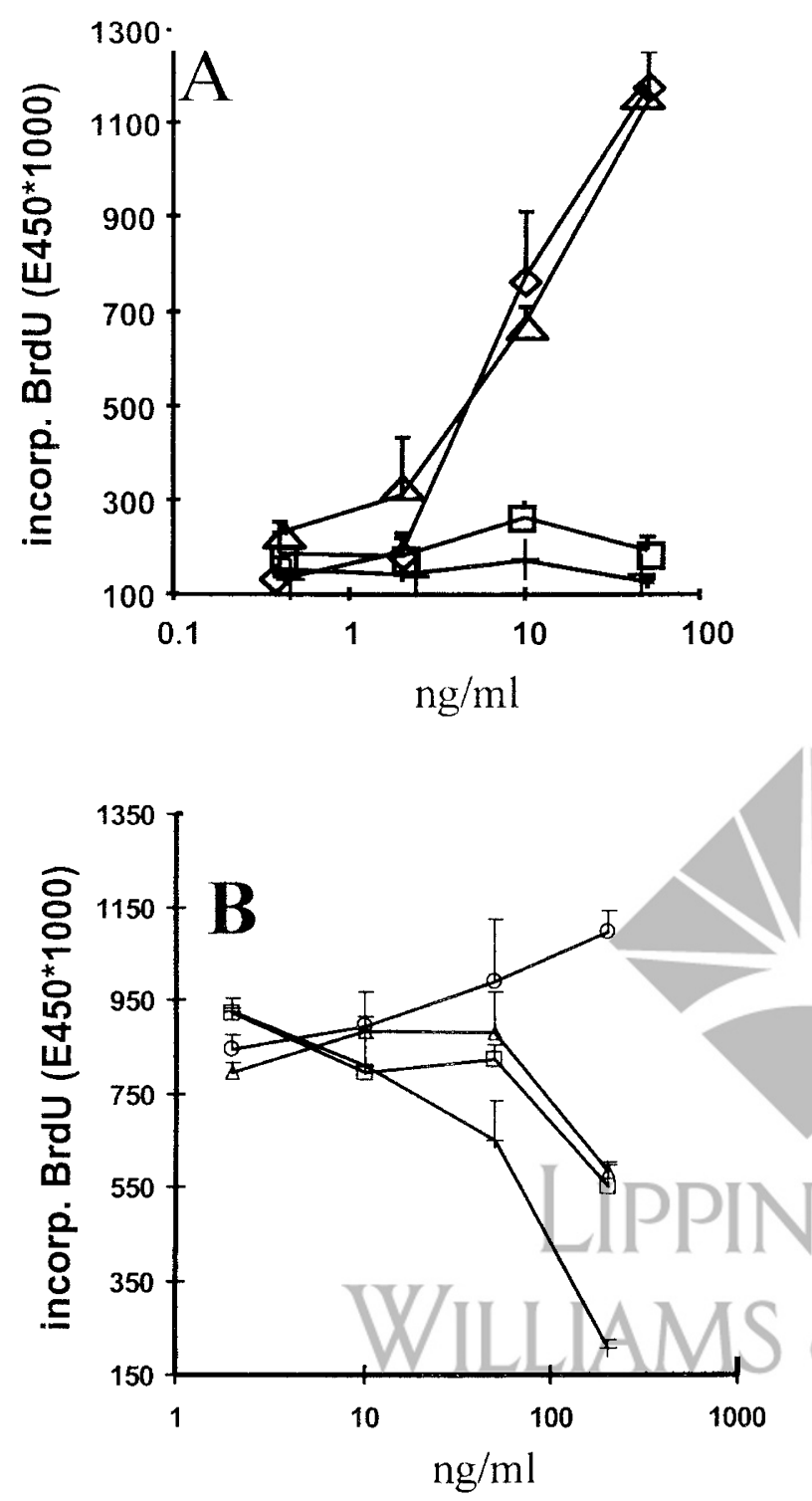
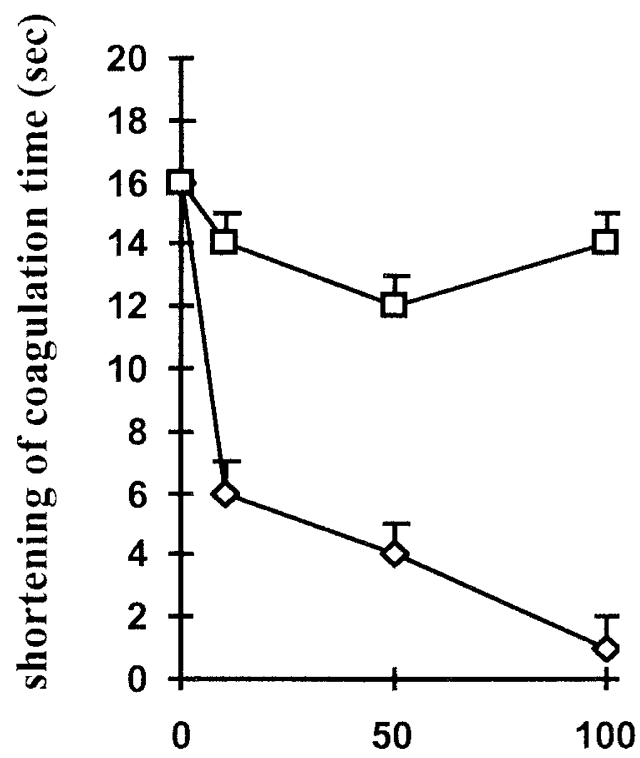

Figure 5.

Specific inhibition of wtVEGF-induced tissue factor (TF) expression by HDVEGF. HUVE cells were incubated with $20 \mathrm{ng} / \mathrm{ml}$ VEGF and increasing concentrations of HD-VEGF $(-\diamond-)$ or VEGF-C1S as a control protein $(-\square-)$. TF-expression was measured in a blood coagulation assay as described in the text. Note that VEGF-C1S had no effect on wtVEGF-induced tissue factor expression, whereas only a 4-fold excess of VEGF-C2SL2/C4SL1 sufficed to block TF expression almost completely.

drium, the layer of cells surrounding the metatarsal. Upon culture, these endothelial cells form PECAM-1 positive tube-like structures, a process which can be stimulated by VEGF and inhibited by neutralizing antiVEGF antibodies (Deckers et al, 2000). HD-VEGF significantly inhibited the formation of the tube-like structures in a dose dependent manner, as demonstrated by PECAM-1 staining (Fig. 7).

\section{Figure 4.}

A, Induction of proliferation of HUVEC by VEGF and VEGF mutants. Experimental procedures are explained in the text. Note that homodimeric VEGF-L2 $(-\triangle-)$ displays an activity similar to that of wtVEGF $(-\diamond-)$, whereas homodimeric VEGF-L1 (- $\square$ ) and HD-VEGF $(-+-)$ do not display detectable activity. B, Specific inhibition of wtVEGF induced HUVEC proliferation by HD-VEGF. HUVE cells were incubated with increasing amounts of HD-VEGF (-+—), VEGF-C4SL1 (一 - -), VEGF-L2 (-○一), or VEGF-C2SL2 $(-\triangle-)$ before activation with $10 \mathrm{ng}$ wtVEGF. Elevated proliferation of cells in response to VEGF-L2 is caused by the proliferation-inducing capacity of this mutant (see also 4A). Values on the Y-axis represent absorption, measured after immunodetection of incorporated BrdU.

because a number of other VEGF-mutants, as well as a mixture of its monomeric subunits ([C2SL2]+[C4SL1]), did not inhibit wtVEGF activity (Fig. 6B).

Finally, to test whether HD-VEGF was able to inhibit angiogenesis, we used an ex vivo fetal bone angiogenesis assay that has previously been shown to depend largely on endogenously produced VEGF (Deckers et al, 2000). When fetal metatarsals are explanted, platelet-endothelial cell adhesion molecule (PECAM)- 1 positive cells are located in the perichon-

\section{Discussion}

In PDGF, three receptor-binding domains designated loop 1,2, and 3 are present (Andersson et al, 1995). We argued that the resemblance in tertiary structure between PDGF and VEGF could be taken further to predict the receptor binding determinants in VEGF, which led to the loop swapping experiments described here. Loop 1 is then defined as ranging from Phe36 to lle46, loop 2 as ranging from Asp63 to Pro70, and loop 3 as ranging from Lys84 to His90 (numbering according to the mature VEGF sequence, Muller et al, 1997). The involvement of loops 2 and 3 in receptor binding were confirmed by alanine-scanning experiments (Keyt et al, 1996). These led to the identification of a positively charged surface in loop 3 , which is essential for binding to KDR/Flk-1 and a negatively charged surface in loop 2, which is responsible for binding to Flt-1 and, to a lesser extent, to KDR/Flk-1 (Fuh et al, 1998; Keyt et al, 1996). In agreement with this latter finding, our VEGF-L2 had an only 3-fold reduced affinity for KDR/Flk-1. 
A

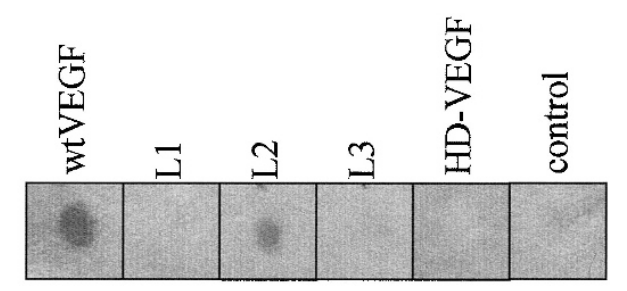

B

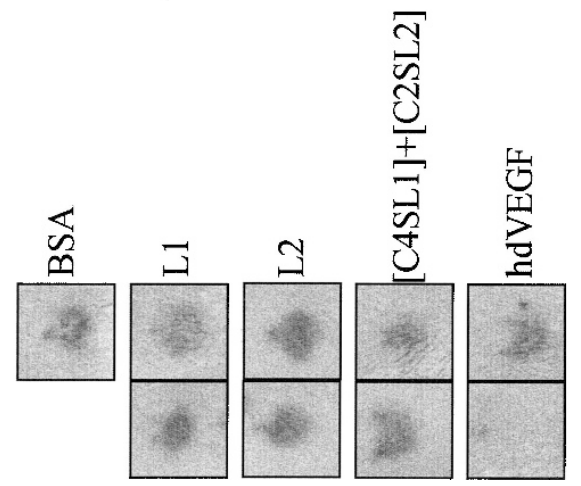

$25 \mathrm{ng}$

$100 \mathrm{ng}$

Figure 6.

Vascular permeability (VP) induction in the Miles assay and its specific inhibition by HD-VEGF. The assay was performed as described in the text. Shown is a representative example of three assays. A, VP-inducing activity of $25 \mathrm{ng}$ of the indicated proteins. L1 = VEGF-L1, L2 = VEGF-L2, L3 = VEGF-L3, control $=\mathrm{DMEM} / \mathrm{BSA}$. Note the complete absence of activity of VEGF-L1, whereas VEGF-L2 showed reduced activity in this assay. The activity of VEGF-L3 at higher concentrations is not shown in this picture. B, Inhibition of wtVEGF-induced VP by VEGF mutants. Guinea pigs were injected intracutaneously with $25 \mathrm{ng}$ or $100 \mathrm{ng}$ of the indicated proteins before injection of $25 \mathrm{ng}$ wtVEGF at the exact spots of the primary injections. L1 = VEGF-L1, L2 = VEGF-L2, [C2SL2]+[C4SL1] represents a 1:1 combination of the monomeric subunits; $100 \mathrm{ng}$ of HD-VEGF, but not of the other (combination of) mutants, inhibited VEGF-induced VP completely. Preinjection of BSA represents a negative vehicle control.
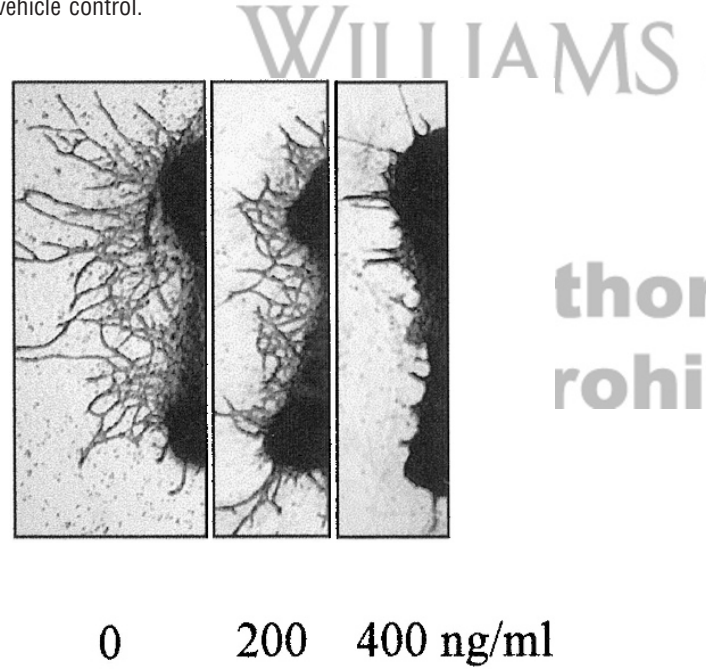

Figure 7.

Effect of HD-VEGF on endothelial sprouting. Fetal mouse metatarsals were cultured in the presence of indicated concentrations of HD-VEGF for 14 days. Shown is a platelet-endothelial cell adhesion molecule (PECAM)-1 staining of metatarsals, cultured under the conditions as described in the text. Note the strong reduction in vascular outgrowth by HD-VEGF.

Previous reports suggesting an involvement of loop 1 in receptor binding are confined to the identification of an interaction between Ile46 and KDR/Flk-1 (Fuh et al, 1998), whereas other mutations in this region had no effect or even led to increased affinity for KDR/ Flk-1 (Keyt et al, 1996).

To dissect the contributions of loops 1 to 3 to VEGF-receptor binding further, we have substituted these domains with the corresponding loops from PDGF-B, alone or in combination with a mutation of one of the cysteines involved in dimerization. Domain swapping of loop 1 with the corresponding domain of PDGF-B led to a complete loss of activity in proliferation assays, procoagulant assays, and in the Miles VP assay. Because VEGF-L1 selectively bound Flt-1 and not KDR, this demonstrates that KDR/Flk-1 and not Flt-1 regulates these activities, at least in HUVEC. This is further supported by our observation that also the VEGF-L3 mutant, in which only KDR/Flk-1 binding is compromised, was unable to up-regulate TF expression and had severely reduced Miles activity (data not shown; and Fig. 6A). The KDR/Flk-1-dependence of TF expression is in agreement with a previous report on VEGF-E, which is a selective ligand for $\mathrm{KDR} / \mathrm{Flk}-1$ and induces TF expression to a similar extent as wtVEGF (Meyer et al, 1999).

Although the affinity of VEGF-L2 for KDR/Flk-1 was decreased only three fold, this mutant displayed diminished activity in the TF assay. Still it had wild-type activity in the proliferation assay as shown in Figure 4. However, we noted that the proliferative activity of VEGF-L2 on HUVEC depended strongly on the batch of HUVEC used, being lower than wild-type VEGF in other batches. It is possible that different receptor expression profiles are responsible for this observation. Higher KDR/Flk-1 expression levels might then compensate for the somewhat lower affinity of VEGF-L2 for KDR/Flk-1 in proliferation assays but not in TF assays.

The affinity of the VEGF-L2 mutant for the KDR/ Flk-1 receptor was only slightly decreased, whereas affinity for the Flt-1 receptor was reduced more than 100-fold. We combined the receptor-selective mutants VEGF-loop 1 and VEGF-loop 2 in a heterodimer, HD-VEGF, with one intact receptor-binding interface and one interface that was predicted to bind neither KDR/Flk-1 nor Flt-1, thus leading to the broadly antagonistic VEGF variant that is displayed in Figure $1 \mathrm{~A}$. Indeed, HD-VEGF inhibited wtVEGF-induced effects in all assays tested. When this work was in progress, Siemeister et al (1998) reported on a heterodimeric VEGF-loop2/loop3 mutant. This heterodimer inhibited VEGF-induced KDR/Flk-1 phosphorylation and endothelial cell proliferation with lower antagonist activity compared to our mutant, because 100 -fold excess was needed to completely block proliferation, whereas ours needed only 20 -fold excess. This difference in potency might be because of the use of a loop 3 swap instead of a loop 1 swap, because in our hands VEGF-L3 had only a slightly decreased affinity for KDR/Flk-1 and showed significant residual activity in the Miles assay. In a recent paper, a similar VEGF-L3 mutant was created (Stacker et al, 1999). In this mutant, the loop 3 domain was swapped with the corresponding domain of PIGF. Interestingly, this mutant showed loss of KDR binding and did not induce 
proliferation but retained a wild-type capacity to induce VP in the Miles assay. Although this result conflicts with other reports on VP regulation, it indicates that the role of the different receptors in VEGF signaling is not yet fully understood. Nevertheless, because VEGF-L1 is inactive in VP assays, a VEGF antagonist mutation of loop 1 is to be preferred above mutation of loop 3.

Neuropilin-I is a co-receptor for the larger VEGF isoforms (Soker et al, 1996). The domain in VEGF that is responsible for neuropilin binding was identified as the basic domain encoded by the exon 7. This gives neuropilin specificity toward the larger VEGF splice variants. Although we have not determined affinities for neuropilin, all our mutants are based on VEGF165 and are thus predicted to have an affinity for neuropilin, similar to that of wtVEGF. Apparently, binding to neuropilin by our mutants can not compensate for the loss of KDR/Flk-1 or Flt-1 binding.

In a recent paper (Dias et al, 2000), it was shown that certain leukemic cell lines and primary leukemias express VEGF as well as KDR/Flk-1 and Flt-1. Interestingly, an anti-KDR monoclonal antibody inhibits VEGF-induced proliferation of these cells, suggesting the existence of an autocrine loop involving the VEGFVEGFR system. VEGF-induced MMP-9 expression and cell migration could only partially be inhibited by this antibody, whereas an anti-Flt-1 antibody had an additive adverse effect on these activities. It is tempting to speculate that HD-VEGF, neutralizing both KDR and Flt-1 receptors, might not only be an antiangiogenic compound, but might also inhibit directly the growth of tumors in which autocrine loops of VEGF-VEGFRs play a role. A potential additional advantage of HD-VEGF is that it might relieve the Flt-1mediated immunosuppressive effect of VEGF (ie, inhibition of tumor-antigen presentation by dendritic cells), resulting in improved anti-tumor immune response. We are currently testing these hypotheses.

In recently published work, a novel KDR-selective VEGF mutant (VEGF-kdrsel) was created that had wild-type affinity for KDR/Flk-1 (Gille et al, 2001; Li et al, 2000), unlike our VEGF-L2 mutant of which the affinity was reduced 3 to 4 times. It will be worthwhile to investigate whether substitution of our VEGFC2SL2 subunit by a VEGF-C2Skdrsel subunit in HDVEGF leads to higher KDR/Flk-1 affinity and thus increased antagonistic potential.

In conclusion, we have created a heterodimeric VEGF variant with one wild-type and one mutated receptor binding interface. This molecule acts as a potent antagonist for both the two VEGF receptors KDR/FIk-1 and Flt-1. Therefore, it should not only inhibit the activities of VEGF, but also the activities of other KDR/Flk-1 or Flt-1 ligands such as PIGF, VEGF-B, C, D, and E. HD-VEGF inhibits wild-type VEGF-activities in a number of in vitro and in vivo assays. Obviously, therapeutic application of such a molecule will require technical solutions to serious potential pitfalls, such as immunogenicity and biologic half-life. We have currently accomplished in vivo expression of HD-VEGF in animals via a gene- therapeutic approach. Studies to test its efficacy as a therapeutic anti-angiogenic agent, not only in tumor treatment but also in other angiogenesis-dependent disorders like rheumatoid arthritis and diabetic retinopathy, are underway.

\section{Materials and Methods}

\section{Construction of Recombinant Baculoviruses}

All enzymes for DNA manipulations and culture media for growth of insect cells were purchased from Life Technologies (Breda, The Netherlands) unless otherwise stated. The isolation and cloning in pBluescript of the coding region of $\mathrm{VEGF}_{165} \mathrm{cDNA}$ has been described previously (Pötgens et al, 1994). Using the helper phage M13K07, single stranded template DNA for mutagenesis was prepared according to standard protocols. Mutagenesis was performed using the Amersham Sculptor Kit (Amersham, Buckinghamshire, England). Oligonucleotides to introduce the C2S and C4S mutations were described previously (Pötgens et al, 1994). The VEGFC1S mutant was created by mutagenesis using oligonucleotide 5'-cgcagctactcccatccaatc-3'. The loop 1 domain was substituted for the corresponding loop of PDGF-B in two sequential steps with the 45-mer B1 (5'-accctggtggacatctcccggcgcctcatagatgagatcgagtac$\left.3^{\prime}\right)$ and the 45-mer B2 (5'-cggcgcctcatagatcgcaccaacgccaacttcaagccatcctgt-3'), using the B1 mutant as a template. This procedure resulted in plasmid pBSVEGF-L1. The loop 2 mutation was introduced using the 51-mer 5'-gggggctgctgcaataaccgcaacgtgcagtgccgccccactgaggagtcc-3', resulting in pBS-VEGF-L2. When required, $\mathrm{C} 2 \mathrm{~S}$ and $\mathrm{C} 4 \mathrm{~S}$ mutations were combined with the $\mathrm{k} 1$ or $\mathrm{L} 2$ mutations to yield clones pBS-VEGFC2SL2 or pBS-VEGF-C4SL1. Inserts were cloned as Sall-Xbal fragments in Sall-Xbal-cut vector pFastBacl (Invitrogen, Carlsbad, California). Recombination of transfer vector and baculovirus genome via directed transposition was allowed to take place in the E.coli strain DH10-BAC. Positive clones, identified by bluewhite screening, were used for isolation of recombinant baculoviral DNA, which was subsequently transfected into SF9 insect cells using Insecticin reagent (InVitrogen). After 5 days, conditioned media were assayed for VEGF (mutant) content by western blotting, using a rabbit anti-VEGF antibody. Viral stocks were generated by infecting SF9 cells at a multiplicity of infection (MOI) of 0.1 and collecting conditioned media 5 days later. To confirm the presence of the desired mutations, DNA was isolated from virus stocks and PCR-sequenced using the Amersham cycle sequencing kit (Amersham). Figure $1 \mathrm{~B}$ shows the amino acid sequences of the mutated regions.

\section{Production and Purification of Mutant VEGF}

SF9 cells were seeded at a density of $10^{5} \mathrm{cells} / \mathrm{cm}^{2}$ in Grace Insect medium, supplemented with $10 \%$ fetal bovine serum and penicillin/streptomycin. Cells were infected with recombinant viruses with an $\mathrm{MOI}$ of 1 . For production of HD-VEGF, SF9 cells were coinfected with VEGF-C2SL2 and VEGF-C4SL1 baculo- 
viruses, both at an $\mathrm{MOI}$ of 5 . After 3 days, medium was collected and mixed with $50 \mu \mathrm{l}$ of heparin-Sepharose slurry/ml (Pharmacia, Uppsala, Sweden). VEGF or its mutants were allowed to bind for 2 hours at $4^{\circ} \mathrm{C}$. After washing with PBS, bound proteins were eluted using $\mathrm{PBS} / 1.5 \mathrm{M} \mathrm{NaCl}$ and stored at $-80^{\circ} \mathrm{C}$. Protein concentrations were measured in a VEGF ELISA (Santa Cruz Biochemicals, Santa Cruz, California). Because mutant VEGFs were not efficiently recognized in this ELISA, concentrations of these were measured by western blotting using a known amount of wtVEGF ${ }_{165}$ as a reference and a rabbit polyclonal anti-VEGF antiserum.

\section{Receptor Binding Assays}

Direct binding of VEGF or mutants thereof to predimerized Flt-1 or KDR was determined by using the extracellular domains of the respective receptors, fused to the Fc region of human IgG1 (KDR-Fc and Flt-1-Fc). Baculoviruses encoding these fusion proteins were a kind gift from Dr. Martiny-Baron, Freiburg, Germany. KDR-Fc or Flt-1-Fc was immobilized on protA-Sepharose (Pharmacia) by incubating $15 \mathrm{ml}$ conditioned SF9-medium, containing approximately 2 $\mu \mathrm{g} / \mathrm{ml} \mathrm{KDR}-\mathrm{Fc}$ or Flt-1-Fc with $0.2 \mathrm{ml}$ protASepharose slurry for 3 hours at $4^{\circ} \mathrm{C}$. Beads were washed twice in PBS and VEGF or mutants were allowed to bind to the immobilized receptors overnight at $4^{\circ} \mathrm{C}$ in binding buffer $(0.1 \% \mathrm{BSA}$ in serum free DMEM). Beads were pelleted and washed three times in PBS. Supernatants and beads (nonbound and bound fractions, respectively) were subjected to electrophoresis through $12 \%$ SDS-PAGE gels. After western blotting, VEGF (mutants) were detected üsing a polyclonal rabbit antiserum (Pötgens et al, 1994).

Alternatively, VEGF (mutants) were tested for their capacity to compete with wtVEGF for binding to cellular receptors as described previously (Clauss et al, 1996). Briefly, monolayers of 3T3-fibroblasts, stably transfected with either full length Flk-1 or Flt-1 cDNA, were incubated with $2 \mathrm{ng}{ }^{125}$-VEGF and increasing amounts of VEGF or VEGF mutants. After binding for 3 hours at $4^{\circ} \mathrm{C}$, cells were washed three times with $\mathrm{PBS} / 0.1 \%$ gelatin, detached with trypsin/EDTA (Gibco, Breda, The Netherlands) and the resulting cell suspension was counted in a gamma counter (Cobra; Canberra Packard, Frankfurt, Germany).

\section{Procoagulant Assay}

Expression of TF by HUVECs in response to VEGF treatment was measured in the procoagulant assay as described previously (Pötgens et al, 1994). In short, HUVECs were incubated in six well dishes (Costar, Acton, Massachusetts) for 5 hours with the agent of study in PMB medium (EMEM, containing 15\% newborn calf serum, $50 \mu \mathrm{g} / \mathrm{ml}$ polymyxin $\mathrm{B}, 2 \mathrm{~mm}$ L-glutamine, and $40 \mu \mathrm{g} / \mathrm{ml}$ gentamycin). Endothelial cells were collected, washed, and suspended in 100 $\mu$ I Veronal buffer (7 mm Veronal, $4 \mathrm{~mm}$ Na-acetate, 130 $\mathrm{mm} \mathrm{NaCl}, \mathrm{pH}$ 7.4). After mixing cells with $100 \mu \mathrm{l} 20 \mathrm{~mm}$
$\mathrm{CaCl}_{2}$ and $100 \mu \mathrm{l}$ pooled normal human plasma at $37^{\circ} \mathrm{C}$, the time needed for fibrin clot formation was recorded. Experiments were always performed in duplicate.

\section{Proliferation Assay}

The proliferative response of HUVE cells toward VEGF or its mutants was measured using a BrdU incorporation kit according to the manufacturer's directions (Boehringer Roche, Mannheim, Germany). HUVE cells were plated at a density of 5000 cells/well in flat bottom 96-well dishes in EMEM containing 10\% pooled human serum and $20 \%$ new born calf serum (NCS; Life Technologies). The next day, medium was replaced by $100 \mu \mathrm{l}$ EMEM containing $0.5 \%$ NCS and factors to be tested. After 1 day of incubation, BrdU was added and cells were cultured further for 1 day. Subsequently, the cells were fixed and incorporated BrdU was quantified by immunodetection according to the manufacturer's protocol.

\section{Miles VP Assay}

Anesthetized Hartley guinea pigs were shaved and injected intramuscularly with $0.2 \mathrm{ml}$ of Phenergan (2.5\% promethazine; Aventis, Strasbourg, France) to reduce mast-cell induced background VP. After 10 minutes, the animals were injected intracardially with 2 $\mathrm{ml}$ of a $0.5 \%$ Evans Blue solution in $0.9 \% \mathrm{NaCl}$. After a further 10 minutes, 25 ng VEGF (mutants) was injected intracutaneously in the flank in a volume of 50 $\mu l$ DMEM containing 1\% BSA. Inhibition of wtVEGFinduced VP was measured by intracutaneous injection of 25 or 100 ng of VEGF mutants, or BSA as a negative control, before injecting $25 \mathrm{ng}$ wtVEGF in the same spots. After extravasation of the Evans Blue dye (10-20 minutes) the animal was photographed. Experiments were performed in triplicate.

\section{Metatarsal Angiogenesis Assay}

Fetuses were removed from 17-day pregnant Swiss 3T3 mice and metatarsals were aseptically dissected (Deckers et al, 2000). The metatarsals (six for each experimental condition) were cultured in 24 wells plates (Greiner, Longwood, Florida) in $150 \mu \mathrm{l} \alpha$-MEM supplemented with $10 \%$ heat inactivated FCS (Integro B.V., Zaandam, The Netherlands) and penicillin/streptomycin (Gibco). After 72 hours, medium was replaced by $250 \mu \mathrm{l}$ fresh medium with or without test compounds. Metatarsals were cultured for a further 14 days with one change of medium after 7 days. Then, cultures were fixed in Zinc-Macrodex formalin (ZnMF) fixative $\left(0.1 \mathrm{M}\right.$ Tris acetate $[\mathrm{pH} 4.5], 0.5 \% \mathrm{ZnCl}_{2}, 0.5 \%$ ZnAcetate, $5 \%$ dextran and 10\% formalin) for 15 minutes at room temperature and subsequently immunostained for PECAM-1 (Deckers et al, 2000). AntiPECAM-1 was a kind gift of Dr. P. Leenen (Erasmus University, Rotterdam, Netherlands). 


\section{Acknowledgements}

We thank Ing Alex Hanssen for technical assistance with the Miles assay experiments and Dr. Georg Martiny-Baron for providing us with the KDR-Fc and Flt-1-Fc baculoviruses.

\section{References}

Achen M, Jeltsch M, Kukk E, Makinen T, Vitali A, Wilks A, Alitalo K, and Stacker S (1998). Vascular Endothelial Growth Factor D (VEGF-D) is a ligand for the tyrosine kinases VEGF receptor 2 (Flk1) and VEGF receptor 3(Flt-4). Proc Natl Acad Sci USA 95:548-553.

Andersson M, Ostman A, Backstrom G, Hellman U, GeorgeNascimento C, Westermark B, and Heldin C-H (1992). Assignment of interchain disulfide bonds in Platelet-Derived Growth Factor (PDGF) and evidence for agonist activity of monomeric PDGF. J Biol Chem 267:11260-11266.

Andersson M, Ostman A, Kreysing J, Bäckström G, van der Poll M, and Heldin C-H (1995). Involvement of loop 2 of Platelet-Derived Growth Factor -AA and -BB in receptor binding. Growth Factors 12:159-164.

Asano A, Yukita A, Matsumoto T, Kondo S, and Suzuki H (1995). Inhibition of tumor growth and metastasis by an immunoneutralizing monoclonal antibody to human Vascular Endothelial Growth Factor/Vascular Permeability Factor Cancer Res 55:5296-5301.

Barleon B, Sozzani S, Zhou D, Weich HA, Mantovani A, and Marmé D (1996). Migration of human monocytes in response to vascular endothelial growth factor (VEGF) is mediated via the VEGF receptor flt-1. Blood 87:3336-3343.

Benjamin LE and Keshet E (1997). Conditional switching of vascular endothelial growth factor (VEGF) expression in tumors: Induction of endothelial cell shedding and regression of hemangioblastoma-like vessels by VEGF withdrawal. Proc Natl Acad Sci USA 94:8761-8766.

Borgström P, Hillan KJ, Sriramarao P, and Ferrara N (1996). Complete inhibition of angiogenesis and growth of microtumors by anti-vascular endothelial growth factor neutralizing antibody: Novel concepts of angiostatic therapy from intravital videomicroscopy. Cancer Res 56:4032-4039.

Cheng S-Y, Huang S, Nagane M, Ji X-D, Wang D, Shih C. C-H, Arap W, Huang C-M, and Cavenee WK (1996). Suppression of glioblastoma angiogenicity and tumorigenicity by inhibition of endogenous expression of vascular endothelial growth factor. Proc Natl Acad Sci USA 93:8502-8507.

Claffey PC, Senger D, and Spiegelman BM (1995). Structural requirements for dimerization, glycosylation, secretion, and biological function of VPF/VEGF. Biochim Biophys Acta 1246:1-9.

Clauss M, Weich H, Breier G, Knies U, Rockl W, Waltenberger J, and Risau W (1996). The vascular endothelial growth factor receptor Flt-1 mediates biological activities. Implications for a functional role of placenta growth factor in monocyte activation and chemotaxis. J Biol Chem 271: 17629-17634.

Deckers MM, Karperien M, van der Bent C, Yamashita T, Papapoulos SE, and Lowik CW (2000). Expression of vascular endothelial growth factors and their receptors during osteoblast differentiation. Endocrinology 141:1667-1674.
Dias S, Hattori K, Zhu Z, Heissig B, Choy M, Lane W, Wu Y, Chadburn A, Hyjek E, Gill M, Hicklin DJ, Witte L, Moore MAS, and Rafii $S$ (2000). Autocrine stimulation of VEGFR-2 activates human leukemic cell growth and migration. J Clin Invest 106:511-521.

Folkman $\mathrm{J}$ and Klagsbrun M (1987a). Angiogenic factors. Science 235:442-447.

Folkman J and Klagsbrun M (1987b). Vascular physiology. A family of angiogenic peptides. Nature 329:671-672.

Fong G-H, Rossant J, Gertsenstein M, and Breitman ML (1995). Role of the Flt-1 receptor tyrosine kinase in regulating the assembly of vascular endothelium. Nature 376:66-70.

Fuh G, Li B, Crowley C, Cunningham B, and Wells JA (1998). Requirements for binding and signaling of the kinase domain receptor for vascular endothelial growth factor. J Biol Chem 273:11197-11204.

Gabrilovitch D, Ishida T, Oyama T, Ran S, Kravtsov V, Nadaf S, and Carbone DP (1998). Vascular endothelial growth factor inhibits the development of dendritic cells and dramatically affects the differentiation of multiple hematopoietic cell lineages in vivo. Blood 92:4150-4166.

Gille H, Kowalski J, Li B, LeCouter J, Moffat B, Zioncheck TF, Pelletier N, and Ferrara N (2001). Analysis of biological effects and signaling properties of Flt-1 (VEGFR-1) and KDR (VEGFR-2). J Biol Chem 276:3222-3230.

Hanahan D and Folkman J (1996). Patterns and emerging mechanisms of the angiogenic switch during tumorigenesis. Cell 86:353-364.

Hiratsuka S, Maru Y, Okada A, Seiki M, Noda T, and Shibuya M (2001). Involvement of Flt-1 tyrosine kinase (Vascular Enodthelial Growth Factor Receptor-1) in pathological angiogenesis. Cancer Res 61:1207-1213.

Joukov V, Pajusola K, Kaipanen A, Chilov D, Lahtinen I, Kukk E, Saksela O, Kalkkinen N, and Alitalo K (1996). A novel vascular endothelial growth factor, VEGF-C, is a ligand for the Flt4 (VEGFR-3) and KDR (VEGFR-2) receptor tyrosine kinases. EMBO J 15:290-298.

Keyt BA, Nguyen HV, Berleau LT, Duarte CM, Park J, Chen H, and Ferrara N (1996). Identification of vascular endothelial growth factor determinants for binding KDR and FLT-1 receptors. Generation of receptor-selective VEGF variants by site-directed mutagenesis. J Biol Chem 271:5638-5646.

Kim KJ, Li B, Winer J, Armanini M, Gillett N, Philips HS, and Ferrara N (1993). Inhibition of vascular endothelial growth factor-induced angiogenesis suppresses tumour growth in vivo. Nature 362:841-844.

Leenders WPJ (1998). Targetting VEGF in anti-angiogenic and anti-tumour therapy: Where are we now? Int J Exp Pathol 79:339-346.

Li B, Fuh G, Meng G, Xin X, Gerritsen ME, Cunningham B, and de Vos AM (2000). Receptor-selective variants of human vascular endothelial growth factor. J Biol Chem 275:2982329828.

Meyer M, Clauss M, Lepple-Wienhues A, Waltenberger J, Augustin HG, Ziche M, Lanz C, Buttner M, Rziha H-J, and Dehio C (1999). A novel vascular endothelial growth factor encoded by Orf virus, VEGF-E, mediates angiogenesis via signalling through VEGFR-2 (KDR) but not VEGFR-1 (Flt-1) receptor tyrosine kinases. EMBO J 18:363-374. 
Millauer B, Longhi MP, Plate KH, Shawver LK, Risau W, Ullrich A, and Strawn LM (1996). Dominant-negative inhibition of Flk-1 suppresses the growth of many tumor types in vivo. Cancer Res 56:1615-1620.

Muller YA, Li B, Christinger HW, Wells JA, Cunningham BC, and de Vos AM (1997). Vascular endothelial growth factor: Crystal structure and functional mapping of the kinase domain receptor binding site. Proc Natl Acad Sci USA 94:71927197.

Neufeld G, Cohen T, Gengrinovitch S, and Poltorak Z (1999). Vascular endothelial growth factor (VEGF) and its receptors. FASEB J 13:9-22.

Olofsson B, Pajusola K, Kaipanen A, von Euler G, Joukov V, Saksela O, Orpana A, Petterson R, Alitalo K, and Eriksson U (1996). Vascular endothelial growth factor B, a novel growth factor for endothelial cells. Proc Natl Acad Sci USA 93:25762581.

Oyama T, Ran S, Ishida T, Nadaf S, Kerr L, Carbone DP, and Gabrilovich G (1998). Vascular Endothelial Growth factor affects dendritic cell maturation through the inhibition of nuclear factor-kB activation in hemopoietic progenitor cells. J Immunol 160:1224-1232.

Park J, Chen H, Winer J, Houck K, and Ferrara N (1994). Placenta growth factor. Potentiation of vascular endothelial growth factor bioactivity, in vitro and in vivo, and high affinity binding to Flt-1 but not to Flk-1/KDR. J Biol Chem 269: 25646-25654.

Pötgens AJ, van Altena MC, Lubsen NH, Ruiter DJ, and de Waal RMW (1994). Covalent dimerization of vascular permeability factor/vascular endothelial growth factor is essential for its biological activity. Evidence from Cys to Ser mutations. J Biol Chem 269:32879-32885.

Shalaby F, Rossant J, Yamaguchi TP, Gertsenstein M, Wu X-F, Breitman ML, and Schuh AC (1995). Failure of bloodisland formation and vasculogenesis in Flk-1-deficient mice. Nature 376:62-66.
Shibuya M (2001). Structure and dual function of vascular endothelial growth factor receptor-1 (Flt-1). Int J Biochem Cell Biol 33:409-420.

Siemeister G, Schirner M, Reusch P, Barleon B, Marmé D, and Martiny-Baron G (1998). An antagonistic vascular endothelial growth factor (VEGF) variant inhibits VEGF-stimulated receptor autophosphorylation and proliferation of human endothelial cells. Proc Natl Acad Sci USA 95:4625-4629.

Soker S, Fidder H, Neufeld G, and Klagsbrun M (1996). Characterization of novel vascular endothelial growth factor (VEGF) receptors on tumor cells that bind VEGF165 via its exon 7-encoded domain. J Biol Chem 271:5761-5767.

Stacker SA, Vitali A, Caesar C, Domagala T, Groenen LC, Nice E, Achen MG, and Wilks AF (1999). A mutant form of vascular endothelial growth factor (VEGF) that lacks VEGF receptor-2 activation retains the ability to induce vascular permeability. J Biol Chem 274:34884-34892.

Takahashi Y, Kitadai Y, Bucana C, Cleary K, and Ellis L (1995). Expression of vascular endothelial growth factor and its receptor, KDR, correlates with vascularity, metastasis, and proliferation of human colon cancer. Cancer Res 55: 3964-3968.

Viglietto G, Maglione D, Rambaldi M, Cerutti J, Romano A, Trapasso F, Fedele M, Ippolito P, Chiappetta G, Botti G, Fusco A, and Persico M (1995). Upregulation of vascular endothelial growth factor (VEGF) and downregulation of placenta growth factor (PIGF) associated with malignancy in human thyroid tumors and cell lines. Oncogene 1:1569-1579 Weng DE and Usman N (2001). Angiozyme: A novel angiogenesis inhibitor. Curr Oncol Rep 3:141-146.

Witte L, Hicklin DJ, Zhu Z, Pytowski B, Kotanides H, Rockwell $P$, and Bohlen $P$ (1998). Monoclonal antibodies targeting the VEGF receptor-2 (Flk1/KDR) as an anti-angiogenic therapeutic strategy. Cancer Metastasis Rev 17:155-161.

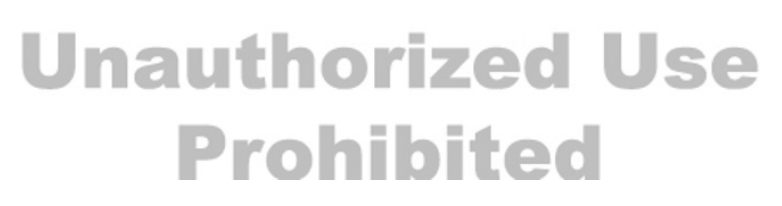

Laboratory Investigation $\bullet$ April $2002 \bullet$ Volume $82 \bullet$ Number 4 